\title{
Evaluating Mechanisms of Change in an Oral Hygiene Improvement Trial With Older Adults
}

Jean Schensul ( $\square$ Jean.schensul@icrweb.org ) Institute for Community Research 2 Hartford Square West, St. 100 Hartford, Ct. 06117

\section{Susan Reisine}

University of Connecticut School of Dental Medicine

Apoorva Salvi

Institute For Community Research

\section{Toan $\mathrm{Ha}$}

University of Pittsburgh Medical Center

James Grady

University of Connecticut School of Medicine

Jianghong Li

Institute For Community Research

\section{Research Article}

Keywords: Oral Health, older adults, clinical trial, prevention, disparities, multilevel, counseling, campaign, intervention mechanisms

Posted Date: January 27th, 2021

DOI: https://doi.org/10.21203/rs.3.rs-127456/v1

License: (c) (i) This work is licensed under a Creative Commons Attribution 4.0 International License. Read Full License

Version of Record: A version of this preprint was published at BMC Oral Health on July 21st, 2021. See the published version at https://doi.org/10.1186/s12903-021-01701-1. 


\section{Abstract}

Objectives. This paper examines the relationship between theoretically-driven mediators and clinical outcomes of a group randomized trial to improve oral health and hygiene of older adults in subsidized housing were compared.

Methods. Six low-income senior residences were paired and randomized into two groups. The first received a face to face counseling intervention (AMI) and the second, a peer-facilitated health campaign (three oral health fairs) both based on Fishbein's Integrated Model. 331 participants were recruited at baseline and 306 completed the post-assessment one month after intervention. Clinical outcome s were Gingival Index (GI) and Plaque score (PS), collected by calibrated dental hygienists. Surveys obtained data on patient background characteristics, and ten oral health beliefs, attitudes, norms and behaviors. GLMM assessed the effects of time, intervention arm, moderators and mediators and intervention by time interactions.

Results. Baseline moderators were similar. Both outcomes improved significantly. GI scores changed from baseline mean of $0.38(s d=.032)$ to $.26(s d=.025)$ and PS scores changed from baseline mean of $71.4(s d=18 \%)$ to $59.1 \%(s d=21 \%)$. Fears, intentionality, norms, worries, flossing and sugar consumption improved significantly in both interventions from baseline to post intervention. Self-efficacy, perceived risk of oral health problems, locus of control and brushing improved significantly only in the counseling intervention. Mechanisms predicting GI improvement were intentionality, locus of control, brushing and flossing in association with the counseling intervention. Mechanisms predicting PS improvement were worries about oral hygiene self-management and fear of oral diseases in association with the AMI intervention. In the trimmed final models, only locus of control (predicting GI) and fears of oral diseases (predicting PS) were significant.

Conclusions. GI and PS improved more in response to the counseling intervention than the campaign. The counseling intervention had a greater impact on mechanisms of change than the campaign. Locus of control, a key concept in oral hygiene interventions including the IM was the main contributing mechanism for GI. Fear, an emotional response drove improvement in PS reinforcing the importance of cognitive/emotional mechanisms in oral hygiene interventions. Improvements in mediators across both interventions suggest a closer examination of the campaign intervention impact on outcomes over time.

Trial Registration: Clinicaltrials.gov NCT02419144, first posted April 17, 2015

\section{Background And Objectives}

Oral health is critical for good general health. WHO (1) and the U.S. Surgeon General $(2,3)$, have called for improving access to oral health treatment and to preventive public health practices. Older adults experience more disparities in both $(4,5)$. Reasons include limited access to quality dental treatment, and few opportunities to experience preventive instruction that would help to prevent caries, periodontal disease and edentulism (6).

There is not yet consensus on what improves oral hygiene, especially in diverse low income older populations. Some general reviews support behavioral interventions (7); others suggest that knowledge, and self-efficacy are critical to achieve successful outcomes (8). Further, there are few detailed descriptions of the components of approaches that show success. Thus additional exploration of motivational and behavioral factors influencing 
oral health and hygiene practices and how they are operationalized as mechanisms of change in oral hygiene interventions is called for.

Many oral health researchers argue that improved theory will identify mechanisms that drive interventions making them more subject to empirical testing $(9,10)$. Recent reviews of oral health interventions suggest that not one, but a number of domains may be important in shaping health outcomes $(11,12)$. The Integrated Model (IM) of health behavior change (13), an approach that incorporates a large number of social, cognitive and behavioral domains, has been shown to be effective in other health related areas (14) and is readily adapted for use in oral health behavior change interventions (15). Fishbein and colleagues address behavior change mechanisms at multiple levels by including social norms and contextual factors in the Integrated Model. They also identify intentionality as the key motivator, bridging other cognitive and behavioral domains leading to behavior change. The significance of intentionality has been questioned by both theoreticians and interventionists, calling for further examination of its role in health-related cognitive-behavioral models $(16,17)$.

The IM model (Figure 1), adapted for oral health behavior change illustrates the cognitive/emotional and behavioral domains (mediators) we have identified as key mechanisms likely to be important in influencing positive oral health clinical outcomes based on the literature and formative research - social norms, beliefs (attitudes, self-efficacy, locus of control), intention and behaviors (sugar consumption, appropriate tooth brushing and appropriate flossing).

Baseline results from our own clinical trial tested the adapted Fishbein model by examining the relationship between these mediators as mechanisms of change and clinical outcomes at baseline. The results showed that intervention mechanisms functioned differently in relation to the two clinical outcomes, gingival index and plaque score. In the baseline analysis, the mechanisms influencing GI were intentionality, locus of control, and more frequent tooth brushing and flossing. The mechanisms influencing PS were fear of oral diseases, locus of control, and worries about managing oral hygiene. While locus of control was common to both, the differences in mechanisms leading to the two outcomes were notable. Direction and control of oral health were characteristic of GI while for PS the driving factors were fear and worries (18)

To address recent literature supporting the importance of multilevel interventions in confronting complex public health problems $(19,20)$, and to evaluate the results of the baseline analyses, the mechanisms included in the model were operationalized into 10 intervention domains and implemented through two different interventions: an individual counseling approach delivered one on one through Adapted Motivational Interviewing (referred to as the AMI); and a group norms change oral health campaign consisting of three oral health fairs delivered by peers to all building residents.

In this clinical trial, two cycle crossover design, in the first cycle one group of 3 buildings received the face-to-face counseling intervention (AMI) and the second group of 3 buildings received the peer led campaign. In the next cycle, each building group received the other intervention. The purpose of the first cycle of the study was to compare one intervention against the other to answer whether the interventions had similar or different outcomes, and to examine possible differences in the predictive role of the mechanisms with respect to each intervention. The purpose of the second cycle was to compare the interactive effects of the two different sequences. This paper concentrates on the first cycle, examining differences between the two interventions in outcomes and mechanisms. 
The paper addresses several key questions: a) did the two interventions result in improvements in the clinical outcomes? b) Did the intervention mechanisms identified and operationalized based on the adapted IM conceptual model change in response to each or both of the interventions? c) Which mechanisms had the greatest impact on the clinical outcomes in each of the two interventions? The study hypothesized that overall the AMI would achieve better outcomes than the campaign because it was intensive, involved multi-model communication around oral hygiene behavior, and was tailored to individuals with explicit attention to the mechanisms. In contrast the campaign, a tailored group intervention, offered overview exposures to the same intervention mechanisms. At the same time, the study design also provided an opportunity to explore how each intervention affected mechanisms of change which in turn had an impact on outcomes.

\section{Methods}

Study Recruitment and Enrolment

Six large rent- subsidized senior apartment buildings in central Connecticut of 125 to 250 apartments were grouped into pairs matched by size and randomized by the study's biostatistician into two groups of three buildings each. A sample size of 123 per group provided $>90 \%$ power to detect mean differences of 0.25 for $\mathrm{GI}$ and 0.66 for PS based on data and SDs from a pilot study, using a 2-group t-test with a two-sided alpha of 0.05 . An attrition rate of $10 \%$ was also assumed. Residents included older adults, aged 62 and above, and people with disabilities under the age of 62 , who were restricted after the first cycle to approximately $30 \%$ of the study population to ensure adequate enrolment of those 62 and over. The study recruited and enrolled 331 participants from these buildings from 2015 - 2017, 175 in the AMI counseling intervention and 157 into the campaign intervention. Of these, 165 completed the $\mathrm{AMI}$ and 76 unduplicated participants attended at least one of the three campaign events based on sign-in registration at the event. Three hundred and six participants completed the T1 assessment. Inclusion criteria included: =>18 years of age, two teeth or more, no conservator; able to comprehend consent form. Exclusion criteria included: temporary building resident; conservatorship; inability to respond correctly to five questions about the study during the consenting process; edentulous; history of infective endocarditis, past six months prosthetic cardiac valve replacement, past six-week myocardial infarction or arterial stent insertion; on dialysis. Eligible candidates signed an informed consent form and completed a baseline (T0) and post intervention (T1) survey and clinical assessment, as well as the intervention assigned to their group. The T1 assessments were administered approximately one month after completion of each intervention.

Ethical Approval and Consent to Participate. The study was reviewed annually by the University of Connecticut Health Center IRB and by NIDCR. All procedures were performed in accordance with the US HHS Belmont Report, 1991, and the Revised Common Rule, 2018 and with requirements of the revised the annually approved study protocol.

Intervention approaches

Intervention activities and processes in both interventions addressed each of the cognitive/emotional and behavioral mediators as mechanisms of intervention in the adapted IM model.

1. The face to face Adapted Motivational Interviewing Intervention (AMI: This 45-60 minute counseling approach was administered by trained bilingual English/Spanish speaking oral health educators no more 
than one month after the baseline survey. It was guided by the IM model. To prepare for the counseling intervention administration, study Pls established cutoff points for scale means in the pre-intervention survey below which it was determined that participants needed intervention(21) Interventionists followed an intervention protocol that began with cognitive/emotional mechanisms, followed by a behavioral intervention instructional component. First, participants were asked to describe their oral health concerns which the interventionist matched with the mechanisms that scored under the cutoff. Next interventionists discussed with participants the mechanisms scoring under the cutoffs and both engaged in a conversation to determine how to best address them, helped by an explanatory script for each mechanism. Next interventionists proceeded to the behavioral instruction component of the intervention. First they reviewed with the participants the pre-intervention plaque scoring record which illustrated in red those teeth had plaque accumulations. This record helped the participant to visualize where to target more effective brushing. The second step involved showing two brief videos in English or Spanish to demonstrate correct brushing and flossing techniques. Then participants practiced brushing and flossing on a typodont (model) and were scored and provided with feedback until they had mastered these activities to the best of their ability. This process was referred to as "practice to mastery" (PM). Finally, with the interventionist, participants created their own plan for oral health improvement with strategies for improving brushing, flossing and selected mechanisms, and kept a copy for themselves. All but one enrollee completed the AMI.

2. The Oral Health Campaign intervention consisted of three oral health fairs held three to four weeks apart, facilitated by a committee of trained peer volunteers in each building with intervention team support. Bilingual campaign committees consisted of 10 - 12 volunteers who did not meet study eligibility criteria and represented a diverse cross-section of residents. The volunteer training program began immediately after the baseline assessment was completed for the entire study sample in each building. It consisted of 12 sessions completed over six to eight weeks (21). Session topics included defining a campaign, team building, oral health and hygiene, and knowledge about all intervention domains (mechanisms) in the IM model and their relationship to the desired clinical outcomes. Once completing these basic instructional sessions, volunteers, with staff support, developed messages for residents based on each of the intervention mechanisms, along with interactive games and other activities. Finally they prepared recruitment strategies and a plan for implementing each of the fairs. Most committee members remained with the campaign for all three oral health fairs. The entire training protocol is posted on the study website (www,projectgoh.com).

Fairs were conducted in English and Spanish simultaneously. The protocol for each fair

included a standard presentation on oral hygiene by dental hygienists delivered in English and Spanish, followed by a question/answer period. Campaign Committee members assisted by project personnel staffed twelve tables, each with a different message associated with a specific mechanism along with related games and informational handouts. Attendees rotated from table to table querying Committee members. At one of the tables, they were instructed on brushing and flossing using a typodont (model). Both enrolled study participants and non-enrolled residents were welcome at the fairs. Attendance was recorded on a sign-in sheet. Each attendee recorded their visit to each table, evaluating their experience with a "passport". Their assignment was to complete the passport before leaving the fair. The passport was turned into project staff on departure from each fair. It served as a record of attendance and provided one measure of dosage. All fairs included a raffle and refreshments. Attendance at each fair averaged 45 people including enrolled participants and visitors and a number of attendees attended 2 or all three fairs. Of enrolled participants $(n=143), 95$ attended at least one fair.

Page 5/28 
Among the anecdotally derived reasons for non-attendance were disability, depression, other obligations (doctor appointments, work schedule), preference for avoiding public events in their building and the incorrect perception that the fair would be conducted in a language they did not understand.

Measures

Most cognitive/emotional domains in the IM model were adapted from pre-existing literature on factors shown to be associated with oral hygiene behavior. Several indices were based on formative research or pilot testing with the study population including fear of oral diseases and worries about oral health self-management. The latter has been validated and published.

Baseline covariates included demographics (age $<61$ vs 62 and older, gender, income $<\$ 900.00$ or $=>\$ 900$, ethnicity (Black non-Hispanic, Hispanic, White non-Hispanic plus other) perceived oral health status rated on a four-point Likert scale as poor (1), fair (2), good (3) or excellent (4), and treated as both a continuous and categorical variable, dichotomized as poor/fair vs good/excellent (22), number of diagnoses that interfered with daily activities ( 0 and 1 or more) and depression measured with the CES-D short form ( $=>4$ high versus $<4$ low) (23).

Cognitive/emotional Mechanisms included oral health self-efficacy: 5 items, (a .603) and locus of control: seven items (a .72) with responses as 4-point Likert scales 1 (low) - 4 (high) for both scales (24); perceived oral health risks (chances of getting specific health problems associated with oral health)(22): five questions with responses rated $1-4$ with 4 as least chances (a .76); fears of oral diseases: 4 items rated $1-4$, with 4 as no fear (a .82); intentionality_(25): a 6 question scale with responses rated as 0 (no intention) to 2 (high intention) (a 72); importance of oral health behavior (26): 9 items rated from 1 not important to 5 important (a .672.); oral hygiene self-management worries scale (OHWSMS): 19 questions with responses rated 1-4 with 4 as least worried (a .93) (27).

Behavioral mechanisms included sugar intake: five questions asking about frequency of consumption of sugar and starch ( 0 is never to 4 as $>$ five times a day); brushing often $(1=<2 /$ day, $2=2+/$ day); and flossing often ( $0=$ $<1 /$ day v. $1=1+$ per day).

Outcome measures consisted of the Gingival Index (GI) (28) and Plaque Score (PS) (29), both assessed by two trained dental hygienists calibrated each year against an experienced dental examiner. The GI assessed the status of gingiva associated with 6 surfaces of each tooth, three buccal and three lingual, by scoring for gingival inflammation from $0=$ no visible inflammation to $3=$ overt inflammation and spontaneous bleeding. The index mean and individual scores were calculated by summing all surface GI scores and dividing by the total number of surfaces. The PS was obtained by the examining hygienist who identified and recorded dichotomous presence or absence scores for bacterial plaque on each of 6 tooth surfaces. PS is expressed as a percentage of surfaces stained red with plaque over total number of surfaces, or a ratio. Reliability of the clinical assessments was assessed prior to T0 and T1. Two hygienists conducted the clinical assessments with the dental director of the study as the gold standard. After training and prior to T1, Kappa improved from a difference of 0.45 to 0.54 to a difference of 0.72 to 1.00 for the Gingival Index and from a difference of 0.46 to 0,78 to a difference of 0.77 to 0.94 for plaque scores. Measures and calibration procedures are further described elsewhere (21) 
Fidelity measures for the AMI counseling intervention included a record of domains covered in each administration, whether the prepared script was utilized, duration of intervention, record of brushing and flossing skills, and a documented plan,. These were reviewed for completeness and accuracy by Pls during each cycle along with reviewing $10 \%$ of audio recordings of AMI administrations in English and Spanish. All participant files included recorded plans. Fidelity measures for the oral health fairs included a record of slide presentations at campaign events, attendance via registration plus passport record of attendance, exposure to each message table, and observations of each campaign.

Statistical Analysis

To investigate within-group change separately by intervention group, paired $t$-tests are reported for clinical outcomes GI and PS (Table 1) and for intervention mechanisms (Table 2). These tests analyze changes from baseline, but are not meant for comparing the two interventions. To make inferential, adjusted assessments of the intervention, we used repeated measures generalized linear mixed models (GLMMs) with main effects of time (0 vs 1), intervention (AMI vs. Campaign), the (time $\times$ intervention) interaction plus covariates of interest (e.g., demographics and health status variables). The interaction terms assess the extent to which the outcomes differ between groups and across time, and interpretations for significant interactions are provided for GI and PS (See Table 4, and Figures 2 and 3). A final reduced model was fit for including significant mediators, moderators and main effects plus interaction for both GI and PS (Table 5). These GLMM models were estimated in the MIXED procedure in SAS version 9.5. For binary outcomes (brushing and flossing) the general estimating equations (GEE) approach was used in the GENMOD procedure in SAS (30). A two-sided level of significance of 0.05 was used to determine statistical significance.

\section{Results}

Approximately $70 \%$ of residents were 62 and above, and most had incomes typically less than $\$ 900.00$ a month. About 50\% were Hispanic, the remainder predominantly Black (24.7\%) and White (9.2\%). Around 73.6\% were combined Medicare and Medicaid insurance recipients. The latter covers basic dental care. Fifty-five percent had been to the dentist in the past year. The majority of those enrolled were living by themselves. Demographics did not differ significantly across building arms at baseline (Table 1).

Both GI and plaque score improved significantly from baseline to first follow-up (mean difference .12, $p>.0001$ ) although the mean differences in improvement were greater in the AMI than in the campaign intervention (see Figures 2 and 3). Gender (female) and education (higher) were significant covariates of improved outcomes overall. While both clinical outcomes improved significantly, the improvement was greater in the AMI than in the campaign intervention group.

Paired T-tests showed that most of the intervention mechanisms improved over time but the mechanisms degree of improvement differed by intervention arm. The AMI counseling intervention resulted in improvements in nine domains and the campaign intervention resulted in improvements in three domains. Where the domain improved for both interventions (fears, intent, norms and worries), the mean difference in improvement in the counseling intervention was greater than the mean difference in improvement for the campaign intervention. Three domains improved only in the counseling intervention group, self-efficacy, perceived risk of oral health problems and 
brushing; one mediator, sugar intake, increased only in the campaign intervention group though the change was trending in the same direction of improvement in the AMI group.

Statistical models (GLMMs) were used to examine moderators and intervention arm as predictors of each mediator. Table 3 addresses the question of whether intervention domain change from T0 to T1, and whether the changes are due to time (from pre to post intervention) or the effects of each or both intervention conditions. Four cognitive domains (perceived risk of oral health problems, fear of oral diseases, intentionality, and worries about oral health self-management) and one behavioral domain, sugar intake, improved over time. Two cognitive domains, self-efficacy and locus of control improved in the AMI counseling intervention only. There were no significant changes in brushing and flossing by building arm, meaning that neither intervention made a difference in brushing and flossing when covariates were controlled for. Important covariates associated with intervention domain directionality were education, age, depression, more than one impairing diagnoses, gender and ethnicity, with variations by domain, as noted in Table 3.

Statistical models (GLMMs) were used to examine each mediator in relation to each clinical outcome indicate where the variable added a significant mediation effect.

Cognitive mechanism improvements that contributed significantly to declines in GI were locus of control and intentionality. Behavioral mechanisms were tooth brushing and flossing frequency. Decreased sugar consumption had borderline significance $(p=.052)$. All of these improvements occurred in association with the counseling intervention. Other mechanisms had no influence on GI. Cognitive mechanism improvements that contributed to declines in PS were fears about oral diseases and worries about oral health self-management which occurred in association with both the AMI and the Campaign interventions.

In the final GLMM model, (Table 5), declines in GI were predicted by improvements in locus of control and declines in PS were predicted by a decrease in fear of oral diseases. Covariates gender (female) predicted reduced GI score, and female gender plus higher education level predicted reduced plaque scores.

\section{Discussion}

This study was conducted with vulnerable older adults between the ages of 50 and 90 living in subsidized senior housing in central Connecticut who received one of two interventions: a one-hour one-time counseling session tailored to individuals, and a building level campaign consisting of three one and one-half hour oral health fairs. The paper focuses on whether oral hygiene outcomes improve as a result of one or the other intervention; whether mechanisms change as a result of each intervention and whether changes in these mechanisms contribute to clinical outcomes associated with each intervention.

Our primary study hypothesis was that the AMI, a counseling intervention, would have a greater effect on clinical outcomes than the campaign, a group norms change intervention. Outcomes improved significantly for both interventions but the AMI contributed to better GI and PS scores compared to the Campaign. A second hypothesis was that the AMI would have a greater effect on study psychological and behavioral mechanisms of change than intervention $B$ because it was more intensive and tailored to individual needs when mechanism scores were below the designated cutoff point. This hypothesis was only partially confirmed. As shown in Table 2 , the mechanisms reflecting more emotion-related domains (fear of oral diseases and worries), intentionality to act and norms (beliefs about the importance of oral health) improved in both interventions. This is not surprising 
since both interventions focused on dispelling myths and misunderstandings about oral health fears and worries and emphasized the importance of oral health action. Brushing improved in the AMI counseling intervention and flossing improved in both interventions but less so in the campaign than in the AMI intervention. An improvement in brushing can be explained by the direct instruction and practice to mastery offered to all participants in the $\mathrm{AMI}$ in contrast to demonstration without practice to mastery in the Campaign intervention. An improvement in flossing in both interventions can be explained by the fact that over half of participants at baseline were not flossing properly or at all and/or were unfamiliar with proper flossing techniques. A greater improvement in AMI than Campaign was likely attributable to the AMI's direct instruction to the participant along with feedback on skills practice. Only the AMI intervention accounted for increases in the related mechanisms of self-efficacy and locus of control. This can be explained by the counseling intervention's focus on gaining control over oral hygiene practice through direct observation and practice to mastery.

The analysis of the relationship between mechanisms and outcomes in our baseline data suggested that improvements in intentionality and locus of control as well as brushing and flossing would result in decreases in Gingival Index. All of these four mechanisms (locus of control, intentionality, brushing and flossing) have been shown in our study to predict GI although only via the AMI counseling intervention. The baseline analysis also predicted that fears and locus of control would be important predictors of PS. Fears has been shown to be a predictor of PS but only via intervention A. Thus the baseline analyses examining mechanisms in relation to clinical outcomes have, for the most part, been borne out by the results of the first round of intervention especially with respect to the AMI counseling intervention. Surprisingly, no behavioral improvements contributed to declines in PS.

As shown in the trimmed GLMM analysis (Table 5) locus of control was the most important predictor of GI. Locus of control over health behaviors is an important construct in oral health $(31,32)$, as well as other preventive health behaviors (33)' and is related to other predictors of improvement in oral hygiene (34). The trimmed model shows that reducing fear of oral diseases is an important contributor to flossing. Our results align with other studies showing the importance of emotional factors such as dental anxiety in oral health treatment (35). Emotional factors are not routinely investigated in oral health preventive behaviors but could provide a new avenue of exploration in future studies. Finally, it is worth noting that though behavioral mechanisms (improved brushing, flossing and sugar intake) were important in the individual GLMM models, none contributed to the final model. In the debate about the relative influence of cognitive and behavioral interventions in oral health, this finding reinforces the importance of including cognitive/emotional components in efforts to improve oral health outcomes.

In terms of covariates, female gender predicted improvements in GI and PS in the final GLMM analysis. More women than men live in senior housing and more women participated in the campaign intervention. Education also contributed to PS. Future interventions with this population should take into consideration both education and health literacy level and make greater efforts to recruit men.

The results suggest that the AMI, a face to face counseling approach, is likely to have a greater impact on clinical outcomes through specific intervention mechanisms than a norms-based oral health campaign with peer facilitation. Though its potential is high, administration has relied on paid professional health educators. With a clearer understanding of which mechanisms are central to improvement and reliance on devices for demonstrating good brushing and flossing practices it may be feasible to train peer educators or health 
professions students to implement Intervention A in a community setting. The curriculum also is transferrable to community based clinic settings. At the same time, improvements were also evident in campaign intervention outcomes, and some mechanisms. Though brushing did not increase, flossing did, suggesting that amplifying the demonstration and practice elements of the oral health campaign could produce equivalent results in community settings such as housing projects, senior centers and other places where older adults congregate. Further, the campaign intervention has been shown to be implementable by trained peer educators with professional support.

\section{Conclusions}

This paper had several primary objectives: first to evaluate the utility of the IM model as the basis for establishing and evaluating intervention mechanisms in two interventions driven by the same conceptual framework; second, to illustrate the role of mediators as mechanisms of intervention in relation to clinical outcomes across the two interventions, and finally to provide results that might help interventionists determine which of the two interventions would be most practical and viable in their settings. The study has shown that the interventions did improve the mechanisms guided by the Integrated Model for oral hygiene improvement, and that most of the mechanisms had an effect on the study clinical outcomes. While it appears that the AMI counseling intervention had the better overall results, the Campaign intervention had unexpected results suggesting that it offers an important and innovative option in introducing oral hygiene into community based settings.

\section{Limitations}

The study was limited to residents of senior housing in central Ct. But $25 \%$ of older low income adults nationally reside in publically subsidized senior housing, making the results potentially generalizable. Not all enrolled participants attended the oral health fairs thus possibly contributing to reductions in effect of mechanisms on the counseling intervention. Finally, limitations in the measurement of behavioral mechanisms or the possibility that there could be intervention domains or mechanisms other than those included in the final models might have had an impact on outcomes.

\section{Abbreviations}

IM - Integrated Model

AMI - Adapted Motivational Interviewing Counseling Intervention

PM - Practice to Mastery

GI - Gingival Index

PS - Plaque Score

T0 - Time Zero - Baseline assessment time point

T1 - Time 1 - Post intervention assessment 


\section{Declarations}

\section{Ethics approval and consent to participate}

IRB Review: The studies were approved annually by the UCONN Health Center IRB, IRB Panel 1 final IRB approval number 14-1990-6.1. The Institute for Community Research IRB deferred to the UCONN Health Center IRB. All participants were read and signed an approved informed consent form. Those few who were illiterate and unable write their signature, signed with an $\mathrm{X}$, which was witnessed by the consenter and an additional witness, both dated and with signatures.

\section{Consent for publication}

Not Applicable

\section{Availability of data and materials}

The datasets generated and/or analyzed during the current study are not publicly available but data used in analyses for the paper are available from the corresponding author on reasonable request.

\section{Competing interests}

The authors declare that they have no competing interests.

\section{Funding}

Funder: National Institute of Dental and Craniofacial Research Grant \# 5U01DE024168. The funders of the study required one change in the approved study design limiting the number of people $<62$ to approximately $30 \%$ of the study. NIDCR also approved reduction of the sample size to 60 people per building after the first study cycle, and the elimination of a non-validated and unstable observational outcome measure from the study. NIDCR Project Officials also reviewed enrollment and retention data monthly from the start of data collection to completion of the last round of data collection and provided a template for reporting. POs and the NIDCR Medical Monitor also reviewed the six month medical monitoring and data quality management reports required for clinical trials and provided feedback to PIs. Rho, a consulting firm assisting NIDCR with clinical trial management, reviewed study IRB/protocol materials, and conducted two onsite audits during the study to review data management and human subjects procedures and provided direct feedback, formal reports and items requiring feedback.

\section{Authors' contributions}

JS conceptualized the paper, wrote all complete drafts of the paper, and guided the data analysis.

SR read, edited and commented on all version of the paper, and made additions to the discussion.

AS conducted all the analyses and created the tables for the study. TH consulted with AS on GLMM analyses. JG as study biostatistician suggested approaches to analysis, advised on all analyses, and reviewed all tables and analysis and results sections. JG reviewed final paper drafts and advised on study language. All authors read and reviewed the final paper.

\section{Acknowledgements}

Page 11/28 
The authors wish to acknowledge the contributions of members of the study team from the University of Connecticut: mauri Barbosa, MSDH, Rita Bodea, BS, Joanna Douglass, DDS, Ruth Goldblatt, DDS., Rajesh Lalla, DDS, PhD; from the Institute for Community Research: Colleen Foster-Bey, MEd. Zahira Medina, MSW. Kim Radda, RN, MA, Eduardo Robles, Lucy Rohena; members of the Oral Health Campaign Committees in each study building; and study participants in each of the study buildings.

\section{References}

1. Petersen PE. Global policy for improvement of oral health in the 21 st century - implications to oral health research of World Health Assembly 2007, World Health Organization. Community Dentistry and Oral Epidemiology. 2009;37(1):1-8.

2. Rollins R, Tomar S, Albino J. 2020 Surgeon General's Report on Oral Health in America: Advances and Challenges Philadelphia, PA.: APHA Meetings, 2019.

3. Satcher D, Nottingham JH. Revisiting Oral Health in America: A Report of the Surgeon General. American Journal of Public Health. 2017;107(S1):S32-S3.

4. Griffin SO, Jones JA, Brunson D, Griffin PM, Bailey WD. Burden of oral disease among older adults and implications for public health priorities. American Journal of Public Health. 2012;102(3):411-8. Epub 2012/03/.

5. Huang DL, Park M. Socioeconomic and racial/ethnic oral health disparities among US older adults: oral health quality of life and dentition. J Public Health Dent. 2015;75(2):85-92. Epub 2014/09/18.

6. Fischer DJ, O'Hayre M, Kusiak JW, Somerman MJ, Hill CV. Oral Health Disparities: A Perspective From the National Institute of Dental and Craniofacial Research. American Journal of Public Health. 2017;107(S1):S36-S8.

7. Watt RG, Daly B, Allison P, Macpherson LMD, Venturelli R, Listl S, et al. Ending the neglect of global oral health: time for radical action. The Lancet. 2019;394(10194):261-72.

8. Schwarzer R, Antoniuk A, Gholami M. A brief intervention changing oral self-care, self-efficacy, and selfmonitoring. British Journal of Health Psychology. 2015;20(1):56-67.

9. Ramaswamy R, Reed J, Livesley N, Kimble L, Boguslavsky V, Garcia-Elorrio E, et al. Unpacking the black box of improvement. International Journal for Quality in Health Care. 2018;30(suppl_1):15-9.

10. Bartholemew, Mullins. Five reasons for theory driven interventions. J Public Health Dent. 2011;71:520-33.

11. Tedesco LA, Keffer MA, Davis EL, Christersson LA. Effect of a Social Cognitive Intervention on Oral Health Status, Behavior Reports, and Cognitions. Journal of Periodontology. 1992;63(7):567-75.

12. McGrath C. Behavioral Sciences in the Promotion of Oral Health. Journal of Dental Research. 2019;98(13):1418-24.

13. Fishbein M. A reasoned action approach to health promotion. Med Decis Making. 2008;28(6).

14. Montaño DE, Kasprzyk D. Theory of Reasoned Action, Theory of Planned Behavior and the Integrated Behavioral Mode. In: Glanz K, Rimer BK, Viwanath K, editors. Health Behavior and Health Education: Theory, Research, and Practice2008. p. 67-96.

15. McCaul KD, O'Neill HK, Glasgow RE. Predicting the performance of dental hygiene behaviors: an examination of the Fishbein and Ajzen Model and self-efficacy expectations. J Appl Soc Psychol. 1988;18(2):114-28. 
16. Liska AE. A Critical Examination of the Causal Structure of the Fishbein/Ajzen Attitude-Behavior Model. Social Psychology Quarterly 1984;47(1):64-71.

17. Schlegel RP, Crawford CA, Sanborn MD. Correspondence and mediational properties of the Fishbein model: An application to adolescent alcohol use. Journal of Experimental Social Psychology. 1977;13(5):421-30.

18. Schensul J, Salvi A, Ha T, Grady J, Li J, Reisine S. Identifying Cognitive/emotional and Behavioral Mediators of Oral Health Outcomes in Vulnerable Older Adults. Jr App Gerontol 2020;Accepted for publication.

19. Agurs-Collins T, Persky S, Paskett ED, Barkin SL, Meissner HI, Nansel TR, et al. Designing and Assessing Multilevel Interventions to Improve Minority Health and Reduce Health Disparities. American Journal of Public Health. 2019;109(S1):S86-S93.

20. Paskett E, Thompson B, Ammerman AS, Ortega AN, Marsteller J, Richardson D. Multilevel Interventions To Address Health Disparities Show Promise In Improving Population Health. Health Affairs. 2016;35(8):142934.

21. Schensul JJ, Reisine S, Grady J. Good Oral Health: An innovative protocol for a community based clinical trial to improve oral health in older adults and people with disabilities. JMIR Research Protocols. 2019.

22. Locker D, Clarke M, Payne B. Self-perceived Oral Health Status, Psychological Well-being, and Life Satisfaction in an Older Adult Population. Journal of Dental Research. 2000;79(4):970-5.

23. Lewinsohn PM, Seeley JR, Roberts RE, Allen NB. Center for Epidemiologic Studies Depression Scale (CES-D) as a screening instrument for depression among community-residing older adults. Psychology and Aging. 1997;12(2):277-87.

24. Sherer M, Maddux JE, Mercandante B, Prentice-Dunn S, Jacobs B, Rogers RW. The Self-Efficacy Scale: Construction and Validation. Psychological Reports. 1982;51(2):663-71.

25. Sanaei Nasab H, Yazdanian M, Mokhayeri Y, Latifı M, Niksadat N, Harooni J, et al. The role of psychological theories in oral health interventions: A systematic review and meta-analysis. International Journal of Dental Hygiene. 2019;17(2):142-52.

26. Brein DJ, Fleenor Jr. TJ, Kim S-W, Krupat E. Using the Theory of Planned Behavior to Identify Predictors of Oral Hygiene: A Collection of Unique Behaviors. Journal of periodontology. 2016;87(3):312-9.

27. Schensul J, Salvi A, Ha T, Reisine S, Jianghong L, Yacihuilca M. Building a New Scale to Measure Worries about Oral Hygiene Self-Management in Vulnrable Older Adults. Gerodontology. 2019;Under review.

28. Löe H, Silness J. Periodontal Disease in Pregnancy I. Prevalence and Severity. Acta Odontologica Scandinavica. 1963;21(6):533-51.

29. O'Leary TJ, Drake RB, Naylor JE. The Plaque Control Record. Journal of Periodontology. 1972;43(1):38-.

30. SAS. Statistical Analysis Software. Cary, NC, USA SAS Institute Inc,.

31. Bryant LL, Quissell DO, Braun PA, Henderson WG, Johs N, George C, et al. A Community-Based Oral Health Intervention in Navajo Nation Head Start: Participation Factors and Contextual Challenges. Journal of Community Health. 2016;41(2):340-53.

32. Hollister MC, Anema MG. Health Behavior Models and Oral Health: A Review. American Dental Hygienists Association. 2004;78(3):6-.

33. Grotz M, Hapke U, Lampert T, Baumeister H. Health locus of control and health behaviour: Results from a nationally representative survey. Psychology, Health \& Medicine. 2011;16(2):129-40. 
34. Newton JT, Asimakopoulou K. Managing oral hygiene as a risk factor for periodontal disease: a systematic review of psychological approaches to behaviour change for improved plaque control in periodontal management. Journal of Clinical Periodontology. 2015;42(S16):S36-S46.

35. Halvari AEM, Halvari H, Deci EL. Dental anxiety, oral health-related quality of life, and general well-being: A self-determination theory perspective. Journal of Applied Social Psychology. 2019;49(5):295-306.

\section{Tables}




\section{Table 1. Participant Characteristics (Covariates) Overall and by Intervention}

Characteristic

Gender

Male

Female

Age $($ Mean $=66.2 ; S D=10.4)$

$<62$

$>=62$

Race/Ethnicity

Hispanic

Black Not Hispanic

White Not Hispanic

and others

Education

Less than high school

More than high school

Marital status

Living alone

Married/with partner

Income (Mean $=\$ 1018 ; \mathrm{SD}=\$ 531.6)$

$<\$ 900$

$>=\$ 900$

Time since last visit to the dentist

$\begin{array}{llll}<1 \text { year } & 55.2 & 55.5 & 57.2 \\ >=1 \text { year } & 43.7 & 44.5 & 42.8 \\ & & & \end{array}$

No. Diagnoses $($ Mean $=3.8(\mathrm{sd}=1.4) ;$ median $=4$
$0-4$
67.4
67.8
32.6
32.2
66.9
$4+$

No. Diagnoses that Interfere with daily

activities (Mean = $1.4(s d=1.0) ;$ Median =0)

42.0

43.1

$1+$

58.0

56.9

40.8

59.2

CES-D-SF $($ Mean $=4.1(\mathrm{sd}=2.2) ;$ Median $=4$

0-4 


\begin{tabular}{|cccc|}
\hline $4+$ & 57.4 & 43.1 & 57.3 \\
\hline Self-Rating of oral health & 42.6 & 56.9 & 42.7 \\
Poor and Fair & & & 65.6 \\
Good and Excellent & 63.1 & 60.9 & 34.4 \\
\hline
\end{tabular}


Table 2, Mean differences over time in Intervention mechanisms for each intervention arm

Domain Intervention A Intervention B

\begin{tabular}{|c|c|c|c|c|c|c|}
\hline $\begin{array}{l}\text { Characteristics } \\
(\mathrm{N}=163)\end{array}$ & Mean (SD) & $\begin{array}{l}\text { Difference } \\
\text { in Mean } \\
\text { (SD) }\end{array}$ & $\begin{array}{l}\text { Paired } \\
\text { t-test } \\
\text { p-value }\end{array}$ & Mean (SD) & $\begin{array}{l}\text { Diff. in } \\
\text { Mean (SD) }\end{array}$ & $\begin{array}{l}\text { Paired } \\
\text { t-test } p \text {. } \\
\text { value }\end{array}$ \\
\hline
\end{tabular}

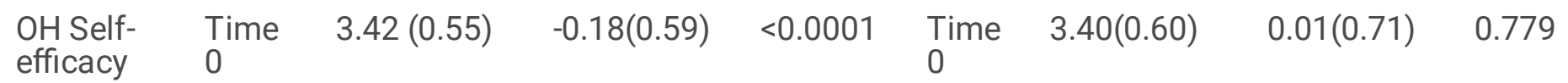

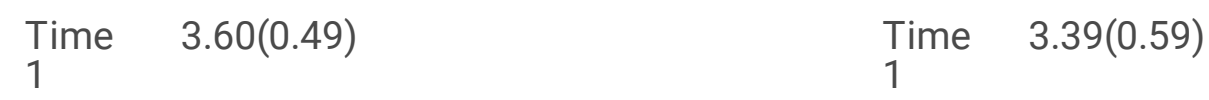

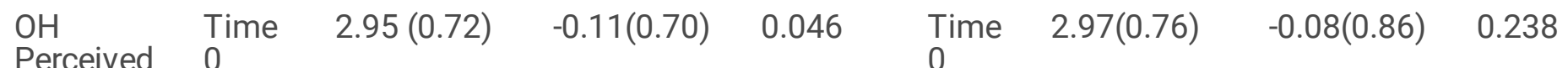

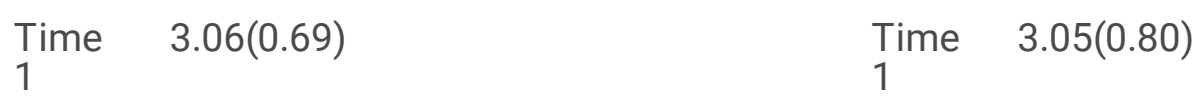

$\begin{array}{llllllll}\mathrm{OH} F & & \end{array}$

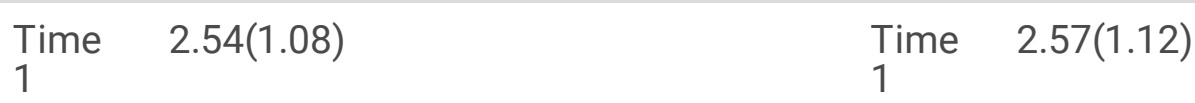

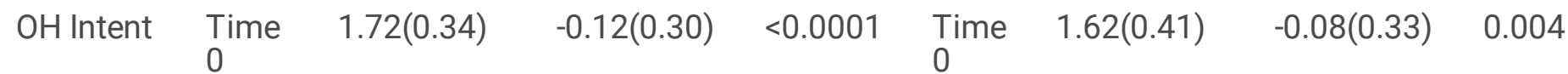

Time 1.84(0.28) $\quad$ Time $1.71(0.37)$

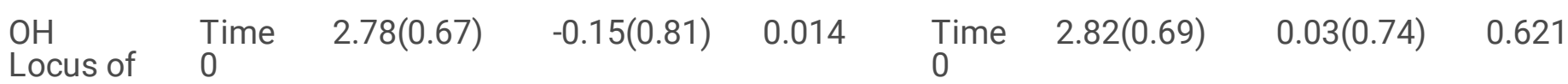
control

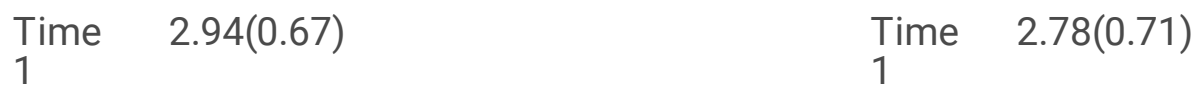

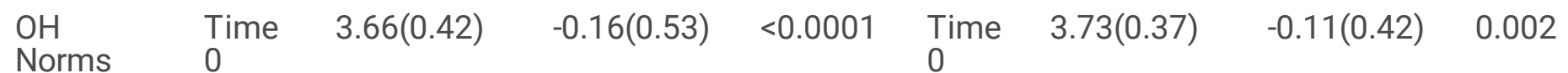

$\begin{array}{llll}\text { Time } & 3.82(0.35) & \text { Time } & 3.84(0.28)\end{array}$

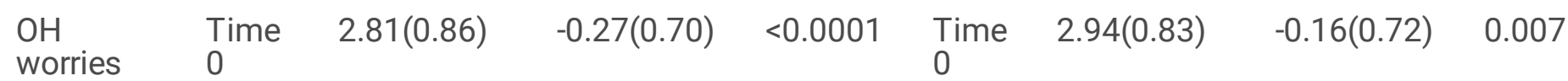

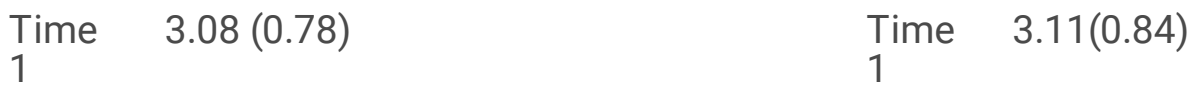

$\begin{array}{lllllllll}\text { Sugar } & \text { Time } & 0.70(0.49) & 0.07(0.47) & 0.052 & \begin{array}{l}\text { Time } \\ 0\end{array} & 0.71(0.48) & 0.15(0.49) & <0.0001\end{array}$

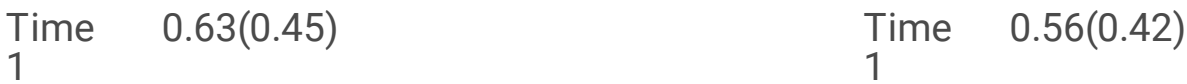

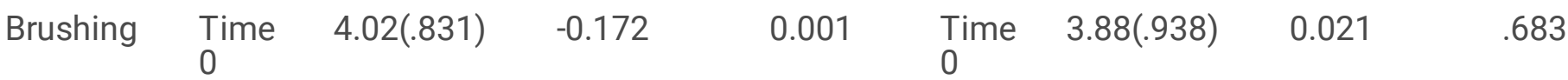

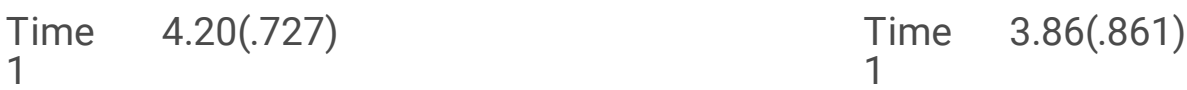




\begin{tabular}{|c|c|c|c|c|c|c|c|c|}
\hline \multirow[t]{2}{*}{ Flossing } & $\begin{array}{l}\text { Time } \\
0\end{array}$ & $2.28(1.880)$ & -.718 & 0.001 & $\begin{array}{l}\text { Time } \\
0\end{array}$ & $1.93(1.75)$ & -.273 & 0.029 \\
\hline & $\begin{array}{l}\text { Time } \\
1\end{array}$ & $2.91(1.534)$ & & & $\begin{array}{l}\text { Time } \\
1\end{array}$ & $2.20(1.730)$ & & \\
\hline
\end{tabular}


Table 3: Moderators, Time and Intervention (A or B) as Predictors of Intervention mechanisms (GLMM)*

Model

1. Self-efficacy

2. Perceived risk

3. Fears of oral health diseases

3. Fears of oral health
diseases

Std.

error

Estimate
Intervention $\times$ time $(A$ with $B$ - as ref)

Race (Hispanics vs

Blacks - as ref)

Income (More than $\$ 900$ vs Less than or equal to $\$ 900$ - as ref)

Age (62 and above vs 62 or less - as ref)

Oral health rating (Good and Excellent vs Poor and Fair - as

ref)
0.199

0.302

$-0.206$
Confidence interval value Upper Lower

0.074

0.007

0.053

0.34

0.17

0.43

$0.066<.0001$

0.17

0.071

0.004

$-0.34$ $-0.06$

$\begin{array}{lllll}-0.206 & 0.071 & 0.004 & -0.34 & -0.06\end{array}$

0.278

0.074

0.0002

0.13

0.42

0.355

0.069

$<.0001$

0.21

0.49
Diagnosis that interfere with daily life ref)

$-0.162$

0.072

0.02

$-0.30$

$-0.01$

\begin{tabular}{llllll}
\hline $\begin{array}{l}\text { CES-D (4+ vas 0-4 - } \\
\text { as ref) }\end{array}$ & -0.135 & 0.071 & 0.05 & -0.27 & 0.005 \\
\hline
\end{tabular}

Time

Time (time 1 vs

Baseline - as ref)

$\begin{array}{lllll}0.245 & 0.084 & 0.003 & 0.080 & 0.41\end{array}$

\begin{tabular}{llllll}
$\begin{array}{l}\text { Age (62 and above vs } \\
62 \text { or less - as ref) }\end{array}$ & 0.293 & 0.105 & 0.005 & 0.085 & 0.500 \\
$\begin{array}{l}\text { Diagnosis that } \\
\text { interfere with daily life } \\
\text { activities (1+ vs 0 - as } \\
\text { ref) }\end{array}$ & -0.250 & 0.102 & 0.015 & -0.45 & -0.04 \\
$\begin{array}{l}\text { CES-D (4+ vas 0-4 - } \\
\text { as ref) }\end{array}$ & -0.197 & 0.100 & 0.051 & -0.39 & 0.001 \\
$\begin{array}{l}\text { Race (Hispanics vs } \\
\text { Blacks - as ref) }\end{array}$ & -0.595 & 0.129 & $<.0001$ & -0.85 & -0.339 \\
\hline
\end{tabular}

\begin{tabular}{lllllll} 
4. Oral health & $\begin{array}{l}\text { Time (time 1 vs } \\
\text { Baseline - as ref) }\end{array}$ & 0.0752 & 0.026 & 0.005 & 0.022 & 0.127 \\
\cline { 2 - 7 } & $\begin{array}{l}\text { Gender (Female vs } \\
\text { Male }- \text { as ref) }\end{array}$ & 0.0805 & 0.034 & 0.0213 & 0.012 & 0.14 \\
\hline $\begin{array}{l}\text { Oral health rating } \\
\text { (Good and Excellent }\end{array}$ & 0.091 & 0.035 & 0.01 & 0.021 & 0.16
\end{tabular}


vs Poor and Fair - as

ref)

$\begin{array}{llllll}\text { Diagnosis that } & -0.101 & 0.036 & 0.006 & -0.173 & -0.028\end{array}$

interfere with daily life

activities (1+ vs 0 - as

ref)

$\begin{array}{llllll}\text { Race (White and } & -0.165 & 0.054 & 0.002 & -0.271 & -0.05\end{array}$

others vs Blacks - as

ref)

5. Locus of control

Intervention $\times$ time $(\mathrm{A}$

0.187

0.090

0.03

0.009

0.36

with $B$ as ref)

$\begin{array}{llllll}\text { Education (Above high } & 0.406 & 0.071 & <.0001 & 0.264 & 0.54\end{array}$

school vs Less than

high school - as ref)

Diagnosis that
interfere with daily life

$\begin{array}{lllll}-0.174 & 0.065 & 0.008 & -0.304 & -0.04\end{array}$

activities (1+ vs 0 - as

ref)

6. Oral health norm-

belief of oral health

hygiene and behavior

$\begin{array}{llllll}\text { Intervention (A with B - } & -0.099 & 0.043 & 0.02 & -0.186 & -0.013\end{array}$

as ref)

$\begin{array}{llllll}\text { Time (1 vs Baseline }- & 0.103 & 0.039 & 0.008 & 0.026 & 0.181\end{array}$

as ref)

Oral health rating

0.061

0.031

0.04

0.0007

0.123

(Good and Excellent

vs Poor and Fair - as

ref)

7. Worries of oral health diseases

Time (1 vs Baseline as ref)

0.18

0.060

$0.001 \quad 0.069$

0.30

Age (62 and above vs

62 or less - as ref)

0.17

0.084

0.03

0.0081

0.34

Diagnosis that

$-0.20$

0.082

0.01

$-0.36$

$-0.040$

interfere with daily life activities (1+ vs 0 - as ref)

\begin{tabular}{llllll|}
$\begin{array}{l}\text { CES-D (4+ vas 0-4 - } \\
\text { as ref) }\end{array}$ & -0.21 & 0.081 & 0.007 & -0.37 & -0.057 \\
$\begin{array}{l}\text { Race (Hispanics vs } \\
\text { Blacks - as ref) }\end{array}$ & -0.45 & 0.104 & $<.0001$ & -0.66 & -0.25 \\
\hline
\end{tabular}

8. Sugar intake

Time ( 1 with baseline - as ref)

$-0.2767$

0.04396

$<.0001$

$-0.3632$

$-0.1902$ 


\begin{tabular}{|c|c|c|c|c|c|c|c|}
\hline & \multirow[t]{2}{*}{ Model } & & \multirow[t]{2}{*}{ Estimate } & \multirow[t]{2}{*}{$\begin{array}{l}\text { Std. } \\
\text { error }\end{array}$} & \multirow[t]{2}{*}{$\begin{array}{l}\mathrm{p}- \\
\text { value }\end{array}$} & \multicolumn{2}{|c|}{$\begin{array}{l}\text { Confidence } \\
\text { Interval }\end{array}$} \\
\hline & & & & & & Upper & Lower \\
\hline & $\begin{array}{l}\text { Brushing } \\
\text { frequency }\end{array}$ & $\begin{array}{l}\text { Intervention * time (A with B - } \\
\text { as ref) }\end{array}$ & -0.7117 & 0.2857 & 0.0127 & -1.2716 & -0.1519 \\
\hline \multirow[t]{4}{*}{9} & & $\begin{array}{l}\text { Gender (Female vs Male - as } \\
\text { ref) }\end{array}$ & -0.480 & 0.226 & 0.03 & -0.92 & -0.03 \\
\hline & & $\begin{array}{l}\text { Income (More than } \$ 900 \text { vs Less } \\
\text { than or equal to } \$ 900-\text { as ref) }\end{array}$ & 0.741 & 0.238 & 0.001 & 0.27 & 1.20 \\
\hline & & $\begin{array}{l}\text { Diagnosis that interfere with } \\
\text { daily life activities ( } 1+\text { vs } 0 \text { - as } \\
\text { ref) }\end{array}$ & 0.703 & 0.247 & 0.004 & 0.21 & 1.18 \\
\hline & & $\begin{array}{l}\text { Race (Hispanics vs Blacks - as } \\
\text { ref) }\end{array}$ & -0.126 & 0.289 & $<.0001$ & -2.33 & -1.17 \\
\hline \multirow[t]{4}{*}{10.} & $\begin{array}{l}\text { Flossing } \\
\text { frequency }\end{array}$ & $\begin{array}{l}\text { Intervention * time (A with B- as } \\
\text { ref) }\end{array}$ & -0.5160 & 0.2455 & 0.0356 & -0.9972 & -0.0347 \\
\hline & & $\begin{array}{l}\text { Gender (Female vs Male - as } \\
\text { ref) }\end{array}$ & -0.567 & 0.178 & 0.001 & -0.91 & -0.21 \\
\hline & & $\begin{array}{l}\text { Diagnosis that interfere with } \\
\text { daily life activities ( } 1+\text { vs } 0 \text { - as } \\
\text { ref) }\end{array}$ & 0.368 & 0.188 & 0.05 & -0.001 & 0.73 \\
\hline & & Time & -0.4216 & 0.1668 & 0.0115 & -0.7485 & -0.0946 \\
\hline
\end{tabular}

*this table reports on only significant preditors. Absence of time and condition for a mediator means nonsignificance.

** Models generated with GEE statistic for categorical variables. 
Table 4: GLMM Analysis of Intervention Mechanisms, Time, Intervention Arm, Covariates and Clinical Outcomes ${ }^{\star \star *}$

\section{Gingival Index}

\begin{tabular}{|c|c|c|c|c|c|}
\hline Variable & Estimate & Std. error & $P$ value & CL & \\
\hline & & & & Upper & Lower \\
\hline Self-efficacy * ** & -0.02182 & 0.01692 & 0.1983 & -0.05512 & 0.01149 \\
\hline Time & -0.07759 & 0.01911 & $<.0001$ & -0.1152 & -0.03998 \\
\hline Intervention*time (A) & -0.07721 & 0.02646 & 0.0038 & -0.1293 & -0.02514 \\
\hline Perceived risk * ** & -0.00143 & 0.01333 & 0.9149 & -0.02765 & 0.02480 \\
\hline Time & -0.07705 & 0.01920 & $<.0001$ & -0.1148 & -0.03928 \\
\hline Intervention*time (A) & -0.08163 & 0.02632 & 0.0021 & -0.1334 & -0.02984 \\
\hline Intent *, ** & -0.09234 & 0.03015 & 0.0024 & -0.1517 & -0.03302 \\
\hline Time & -0.06996 & 0.01945 & 0.0004 & -0.1082 & -0.03169 \\
\hline Intervention*time (A) & -0.07742 & 0.02656 & 0.0038 & -0.1297 & -0.02515 \\
\hline Norms *, ** & -0.03425 & 0.02415 & 0.1572 & -0.08177 & 0.01327 \\
\hline Time & -0.07338 & 0.01936 & 0.0002 & -0.1115 & -0.03527 \\
\hline Intervention * time $(\mathrm{A})$ & -0.08009 & 0.02637 & 0.0026 & -0.1320 & -0.02821 \\
\hline Locus of control $\left(^{(}\right)$ & -0.04627 & 0.01366 & 0.0008 & -0.07314 & -0.01940 \\
\hline Time & -0.07807 & 0.01906 & $<.0001$ & -0.1156 & -0.04057 \\
\hline Intervention*time (A) & -0.07298 & 0.02629 & 0.0058 & -0.1247 & -0.02125 \\
\hline Worry*, ** & -0.01702 & 0.01342 & 0.2056 & -0.04342 & 0.009383 \\
\hline Time & -0.07398 & 0.01932 & 0.0002 & -0.1120 & -0.03595 \\
\hline Intervention`time (A) & -0.08018 & 0.02633 & 0.0025 & -0.1320 & -0.02836 \\
\hline Fears * ** & -0.01006 & 0.009907 & 0.3107 & -0.02956 & 0.009435 \\
\hline Time & -0.07467 & 0.01932 & 0.0001 & -0.1127 & -0.03666 \\
\hline
\end{tabular}




\begin{tabular}{|c|c|c|c|c|c|}
\hline Intervention * time $(\mathrm{A})$ & -0.08017 & 0.02636 & 0.0026 & -0.1320 & -0.02830 \\
\hline Sugar Intake*, ** & 0.009035 & 0.01961 & 0.6454 & -0.02956 & 0.04763 \\
\hline Time & -0.07468 & 0.01992 & 0.0002 & -0.1139 & -0.03549 \\
\hline Intervention * time $(A)$ & -0.08229 & 0.02636 & 0.0020 & -0.1342 & -0.03043 \\
\hline Brushing * ** & -0.06488 & 0.02670 & 0.0157 & -0.1174 & -0.01234 \\
\hline Time & -0.07580 & 0.01924 & 0.0001 & -0.1137 & -0.03794 \\
\hline Intervention * time $(\mathrm{A})$ & -0.07593 & 0.02652 & 0.0045 & -0.1281 & -0.02375 \\
\hline Flossing *, ** & -0.05892 & 0.02018 & 0.0038 & -0.09863 & -0.01921 \\
\hline Time & -0.07127 & 0.01926 & 0.0003 & -0.1092 & -0.03338 \\
\hline Intervention * time $(A)$ & -0.07495 & 0.02640 & 0.0048 & -0.1269 & -0.02300 \\
\hline \multicolumn{6}{|l|}{ Plaque scores } \\
\hline \multirow[t]{2}{*}{ Variable } & Estimate & Std error & $P$ value & $\mathrm{CL}$ & \\
\hline & & & & Upper & Lower \\
\hline Self efficacy*,** & 0.003714 & 0.01318 & 0.7783 & -0.02222 & 0.02965 \\
\hline Time & -0.08234 & 0.01732 & $<.0001$ & -0.1164 & -0.04825 \\
\hline Intervention *time $(\mathrm{A})$ & -0.08175 & 0.02391 & 0.0007 & -0.1288 & -0.03469 \\
\hline Perceived risk * ** & -0.01705 & 0.01035 & 0.1005 & -0.03742 & 0.003318 \\
\hline Time & -0.08099 & 0.01732 & $<.0001$ & -0.1151 & -0.04691 \\
\hline Intervention*time (A) & -0.08052 & 0.02373 & 0.0008 & -0.1272 & -0.03383 \\
\hline Intent *, ** & -0.00157 & 0.02170 & 0.9422 & -0.04427 & 0.04112 \\
\hline Time & -0.08229 & 0.01739 & $<.0001$ & -0.1165 & -0.04807 \\
\hline Intervention*time (A) & -0.08091 & 0.02377 & 0.0008 & -0.1277 & -0.03412 \\
\hline Norms *, ** & -0.02574 & 0.01887 & 0.1737 & -0.06287 & 0.01140 \\
\hline Time & -0.07957 & 0.01745 & $<.0001$ & -0.1139 & -0.04523 \\
\hline
\end{tabular}




\begin{tabular}{|llllll|}
\hline Intervention * time (A) & -0.07977 & 0.02378 & 0.0009 & -0.1266 & -0.03296 \\
\hline Locus of control *, ** & -0.01988 & 0.01068 & 0.0638 & -0.04090 & 0.001148 \\
\hline Time & -0.08273 & 0.01731 & $<.0001$ & -0.1168 & -0.04866 \\
\hline Intervention*time (A) & -0.07728 & 0.02383 & 0.0013 & -0.1242 & -0.03038 \\
\hline & & & & & \\
\hline Worry * ** & -0.02246 & 0.009910 & 0.0241 & -0.04197 & -0.00296 \\
\hline Time & -0.07807 & 0.01742 & $<.0001$ & -0.1123 & -0.04379 \\
\hline Intervention*time (A) & -0.07914 & 0.02377 & 0.0010 & -0.1259 & -0.03236 \\
\hline & & & & & \\
\hline Fears *,* & -0.02562 & 0.007592 & 0.0008 & -0.04056 & -0.01068 \\
\hline Time & -0.07602 & 0.01720 & $<.0001$ & -0.1099 & -0.04217 \\
\hline Intervention*time (A) & -0.07718 & 0.02348 & 0.0011 & -0.1234 & -0.03097 \\
\hline & & & & & \\
\hline Sugar intake *** & -0.01819 & 0.01419 & 0.2009 & -0.04611 & 0.009737 \\
\hline Time & -0.08746 & 0.01771 & $<.0001$ & -0.1223 & -0.05260 \\
\hline Intervention * time A? & -0.07973 & 0.02371 & 0.0009 & -0.1264 & -0.03307 \\
\hline & -0.01959 & 0.01515 & 0.1967 & -0.04940 & 0.01021 \\
\hline Brushing *** & -0.08044 & 0.01739 & $<.0001$ & -0.1147 & -0.04622 \\
\hline Time & -0.07872 & 0.02383 & 0.0011 & -0.1256 & -0.03182 \\
\hline Intervention* time (A) & -0.07882 & 0.02382 & 0.0011 & -0.1257 & -0.03194 \\
\hline & & & & -0.06155 & 0.01365 \\
\hline Flossing *** & -0.08191 & 0.01733 & $<.0001$ & -0.1160 & -0.04782 \\
\hline Intervention* time (A) & & & & & \\
\hline
\end{tabular}

*refers to sex as covariate; $* *$ refers to education as covariate ***; Slicing effects show that the change occurs in intervention $A$ versus $B$ across all analyses. 


\begin{tabular}{|c|c|c|c|c|c|}
\hline \multicolumn{6}{|l|}{ Gingival Index } \\
\hline \multirow[t]{2}{*}{ Effect } & \multirow[t]{2}{*}{ Estimate } & \multirow[t]{2}{*}{$\begin{array}{l}\text { Standard } \\
\text { Error }\end{array}$} & \multirow[t]{2}{*}{$\operatorname{Pr}>|t|$} & \multicolumn{2}{|l|}{$\mathrm{CL}$} \\
\hline & & & & Lower & Upper \\
\hline Change over time & -0.063 & 0.020 & 0.002 & -0.104 & -0.023 \\
\hline Change in time $\mathrm{x}$ intervention ( $\mathrm{A}$ as reference) & -0.060 & 0.027 & 0.026 & -0.114 & -0.007 \\
\hline Gender (Female vs Male - as reference) & -0.067 & 0.026 & 0.012 & -0.120 & -0.015 \\
\hline Locus of control & -0.037 & 0.013 & 0.007 & -0.065 & -0.010 \\
\hline \multicolumn{6}{|l|}{ Plaque Score } \\
\hline \multirow[t]{2}{*}{ Effect } & Estimate & $\begin{array}{l}\text { Standard } \\
\text { Error }\end{array}$ & $\operatorname{Pr}>|t|$ & $\mathrm{CL}$ & \\
\hline & & & & Lower & Upper \\
\hline Change over Time/ & -0.080 & 0.019 & $<.0001$ & -0.116 & -0.044 \\
\hline Change in Time $x$ Intervention ( $A$ as reference) & -0.067 & 0.024 & 0.005 & -0.115 & -0.019 \\
\hline Gender (Female vs Male - as reference) & -0.038 & 0.017 & 0.03 & -0.072 & -0.003 \\
\hline $\begin{array}{l}\text { Education (More than high school vs Less than high } \\
\text { school - as reference) }\end{array}$ & -0.089 & 0.020 & $<.0001$ & -0.130 & -0.049 \\
\hline Fear of oral diseases & -0.021 & 0.009 & 0.026 & -0.040 & -0.002 \\
\hline
\end{tabular}

\section{Figures}




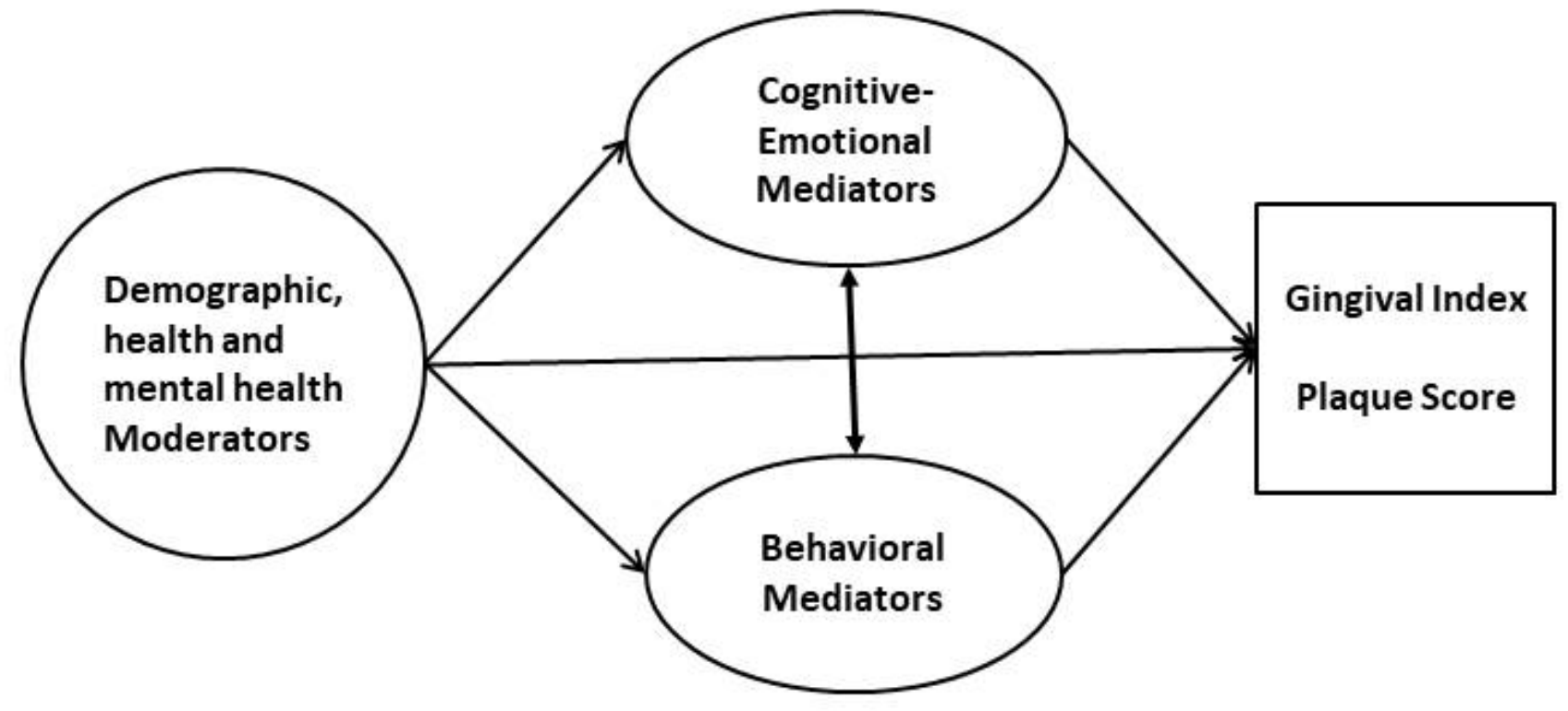

Figure 1

Integrated Model of Behavior Change adapted for Oral Health 


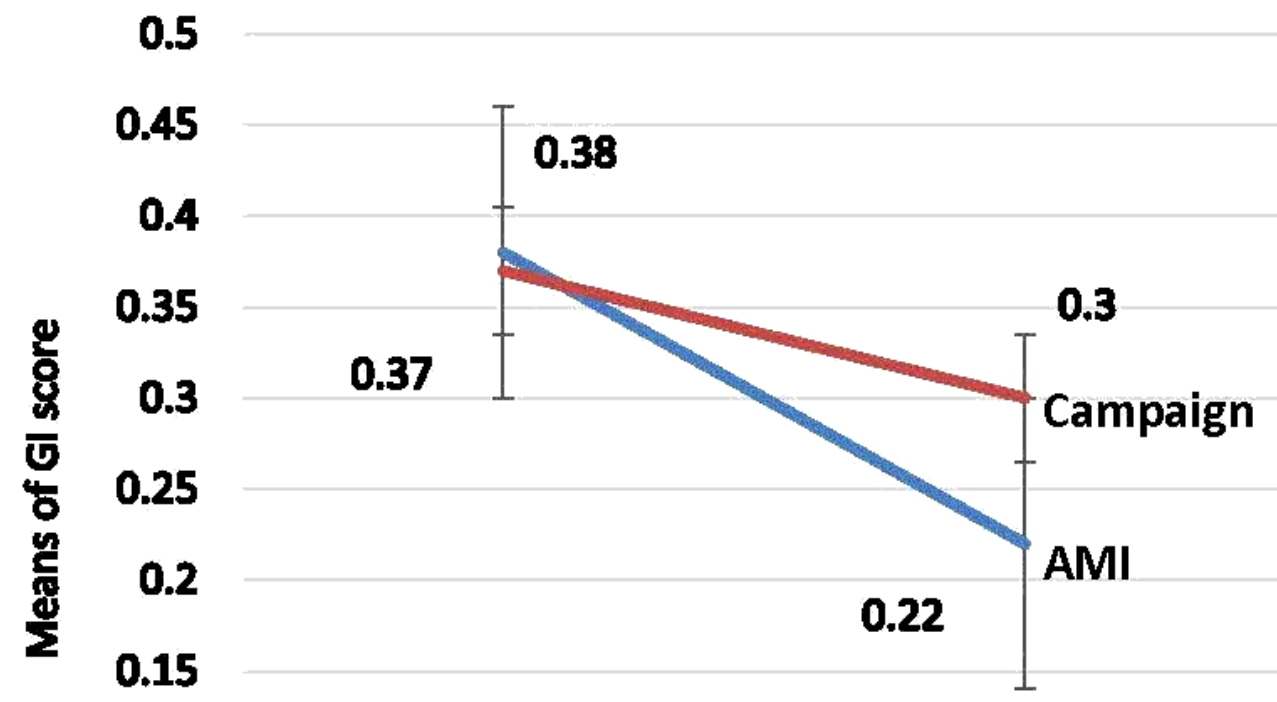

0.1

0.05

0

Time 0

Time 1

Time point by Bullding arm

Figure 2

Changes in Gingival Index Score from T0 to T1 


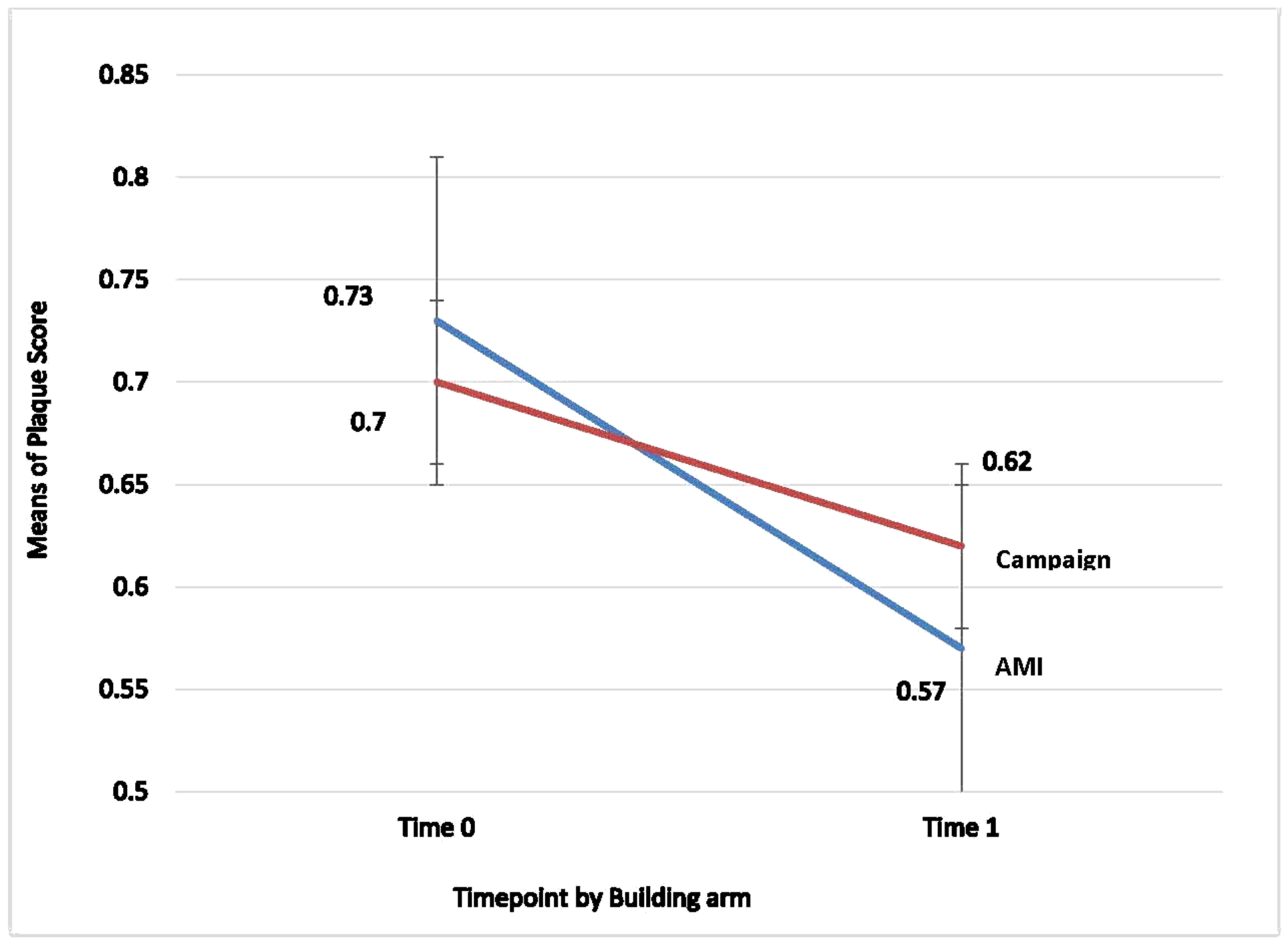

Figure 3

Changes in Plaque Score from T0 to T1 